\title{
miR-4454 up-regulated by HPV16 E6/E7 promotes invasion and migration by targeting ABHD2/NUDT21 in cervical cancer
}

Hui Wang ( $D$ 027whwh@sina.com )

The Central Hospital of Wuhan

Hui Hu

The Central Hospital of Wuhan

Zhenzhao Luo

The Central Hospital of Wuhan

Shuiyi Liu

The Central Hospital of Wuhan

Wangze Wu

The Central Hospital of Wuhan

Man Zhu

The Central Hospital of Wuhan

Jing Wang

The Central Hospital of Wuhan

Yingle Liu

Wuhan University

Zhongxin Lu

The Central Hospital of Wuhan

Research article

Keywords: Cervical Cancer; HPV16 E6/E7; miR-4454; ABHD2; NUDT21

Posted Date: May 6th, 2019

DOI: https://doi.org/10.21203/rs.2.9472/v1

License: (c) (i) This work is licensed under a Creative Commons Attribution 4.0 International License.

Read Full License

Version of Record: A version of this preprint was published at Bioscience Reports on September 1st, 2020. See the published version at https://doi.org/10.1042/BSR20200796. 


\section{Abstract}

The abnormal expression of HPV16 E6/E7 activates oncogenes and/or inactivates tumor suppressor genes, resulting in the selective growth and malignant transformation of cancer cells. miR-4454 was selected by sequencing due to its abnormal high expression in HPV16 E6/E7 positive CaSki cell compared with HPV16 E6/E7 negative C33A cell. Overexpression of miR-4454 enhances cervical cancer cell invasion and migration. ABHD2 and NUDT21 is identified as a target gene of miR-4454.The effects of ABHD2 and NUDT21 on migration and invasion of CaSki and C33A cells were determined. The dual luciferase and RT-qPCR assays confirmed that miR-4454 might regulate its targets ABHD2 and NUDT21 to promote the proliferation, invasion and migration, whereas, inhibit the apoptosis in CaSki and C33A cells.

\section{Introduction}

The incidence of cervical cancer ranks the second among all female tumors with nearly 500,000 women worldwide diagnosed with cervical cancer every year, nearly half of whom ultimately die of the disease 1,2. It has been confirmed that cervical cancer is associated with high-risk human papillomavirus (HPV) infection, with HPV16 and HPV18 being the most common infectious agents found in $99.7 \%$ of cases of cervical squamous cell carcinoma and $94-100 \%$ of cases of cervical adenocarcinoma.1,3-6. China's morbidity and mortality associated with cervical cancer account for nearly one-quarter of the world's total, representing a serious threat to women's health.

In HPV-positive cervical cancers, all of the malignant cells contain at least one copy of the viral genome with transcriptional activity7,8. Recent studies have shown that non-coding microRNAs (miRNAs) are also regulated by E6 and $\mathrm{E} 7$ at the transcriptional and post-transcriptional level,9-13.

In this study, we investigated the molecular mechanism of HPV16 E6/E7-regulating miRNA in cervical cancer progress. We used HPV16 E6/E7-positive human cervical cancer cell line CaSki as the major cell model. The aim of this study was to elucidate the biological functions and potential target of miR-4454 in cervical cancer cells and provide evidence that miR-4454 may serve a potential candidate for the clinical treatment for cervical cancer.

\section{Materials And Methods}

\section{Cell culture}

C33A cell [American Type Culture Collection (ATCC), Rockville, MD, USA], which is an HPV16 E6/E7negative cell line, were maintained in $10 \mathrm{ml}$ of $90 \%$ Eagle's minimum essential medium ( Gibco, Thermo Fisher Scientific, Inc., MA, USA) containing 10\% fetal bovine serum (FBS) (Gibco, USA). The CaSki cell (ATCC, Rockville, MD, USA), which is an HPV16 E6/E7-positive cell line, were maintained in $10 \mathrm{ml}$ of $90 \%$ RPMI-1640 medium (Gibco, USA) containing 10\% FBS (Gibco, USA). After mixing, $1 \mathrm{ml}$ of the medium was added to each well of the culture plate and placed in a 5\% CO2, 37(C incubator. 


\section{The differentially expressed gene was selected by sequencing}

siRNA sequences are synthesized by Sangon Biotech (Shanghai) Co., Ltd. sh-E6: F:5'GGAGCGACCCAGAAAGUUATT-3',R:5'-UAACUUUCUGGGUCGCUCCTT-3'; Then the interfering shRNA was screened and transfected into CaSki cell. Finally, CaSki cell and siRNA cell were sent to the personalbio (Shanghai, China) for sequencing and screening for differential miRNA. Sample processing: After total RNA was extracted, the RNA quality was detected. Then the small RNA library was constructed by IlluminatheTruSeq Small RNA Sample prep Kit. The enriched library was amplified by PCR and purified by gel electrophoresis. The library quality was checked by Agilent High Sensitivity DNA Kit using Agilent 2100 Bioanalyzer. After testing the qualified library, the library was quantified by Quant-iT Picogreen dsDNA Assay Kit. Finally, single strand library was used as template to bridge PCR amplification, and primers were annealed and sequenced.Data analysis: The original data is de-joined and filtered, and the filtered sequence is de-re-processed. The deleted small RNA sequence is annotated on the basis of the reference genome and the corresponding annotation abundance is given. After statistical analysis, the differentially expressed miRNAs were clustered and predicted, and the predicted target genes were enriched and analyzed.

\section{Construction of miR-4454 mimics and miR-4454 inhibitor}

The sequence of the hsa-miR-4454 mature body is 5'-GGAUCCGAGUCACGGCA CCA-3' (http://www.mirbase.org/cgi-bin/mature.pl?mature_acc=MIMAT0018976), which was synthesized by GenScript (Nanjing) Co., Ltd. (Nanjing, Jiangsu, China). The miR-4454 mimics/inhibitor (RIBOBIO, Guangzhou, China) was then successfully constructed based on this sequence.

\section{Construction of pre-ABHD2/NUDT21 and sh-ABHD2/NUDT21 plasmids}

The template strand sequences were as follows: ABHD2 positive-sense 5 'GATCGCCAATGGGAGCGTAACAAGTTTCAAGAGAACTTGTTACGCTCCCATTGGCTTTTTT-3',antisense:5'AATTAAAAAAGCCAATGGGAGCGTAACAAGTTCTCTTGAAACTTGTTACGCTCCCATTGGC3';NUDT21 positive-sense:5'-

GATCGCGCATGAGGGAAGAATTTGATTCAAGAGATCAAATTCTTCCCTCATGCGCTTTTTT-3', antisense:5'AATTAAAAAAGCGCATGAGGGAAGAATTTGATCTCTTGAATCAAATTCTTCCCTCATGCGC-3'. The small hairpin RNA (shRNA) sequences were then obtained by polymerase chain reaction (PCR) amplification, and inserted in the vector plasmid pSIREN connected by EcoRI and BamHI enzymes (Thermo Fisher, MA, USA). Finally, the recombinant plasmids pSIREN-shRNA-ABHD2 and pSIREN-shRNA-NUDT21 were amplified and infected into the CaSki and C33A cells. The template strand sequence of ABHD2 was as follows: forward primer 5'-CGCGGATCCATGAATGCCATGCTG-3', reverse primer 5'-CCGCTCGAGTCACT CCAGGTCGGCCT-3'. The vector plasmid pcDNA3.1 was digested and connected by EcoRI and Xhol enzymes (Thermo Fisher, MA, USA). The recombinant plasmids pcDNA3.1-pre-ABHD2 and pcDNA3.1-preNUDT21 were amplified and infected into the CaSki and C33A cells. The template strand sequence of ABHD2 was as follows: forward primer 5'-CGCGGATCCATGTCTGTGGTAC CG-3', reverse primer 5'CCGCTCGAGTCAGTTGTAAATAAAATTG-3'.' 


\section{Western blot}

Total protein were extracted by RIPA buffer(Beyotime, Shanghai, China) involved in protease inhibitors.

The protein concentration measured by BCA protein assay kit (Thermo Fisher Scientific). The supernatant soluble lysate was mixed with loading buffer and the solution boiled for $10 \mathrm{~min}$ and stored at $-20^{\circ} \mathrm{C}$. Isolated proteins were electrophoresed on 8\% SDS-PAGE electrophoresisand transferred to a $0.22-\mu \mathrm{m}$ PVDF membrane and blocked with $5 \%$ nonfat milk. Afterward, the membranes were then incubated with primary antibodies against GAPDH (1:5000, ab181602, Abcam, Shanghai, China), ABHD2 (1:1000, ab23041, Abcam, Shanghai, China), NUDT21(1:3000, ab183660, Abcam, Shanghai, China) overnight at $4^{\circ} \mathrm{C}$. The membranes were then incubated with the HRP-conjugated secondary antibody $(1: 10,000$, Santa Cruz) and the protein level detected by chemiluminescence .

\section{Transwell assay}

A transwell assay was performed to investigate the migration and invasion of C33A and CaSki cells. The cells were harvested for $24 \mathrm{~h}$ before cell suspension, and then the cells were digested to be used as signal cells at a $5 \times 105 / \mathrm{ml}$ concentration. The cells were suspended using a serum-free medium containing bovine serum albumin, and $100 \mu$ of the cell suspension was added to the transwell chamber. The 24well plate chamber was filled with $600 \mu \mathrm{l}$ of medium per well containing $10 \% \mathrm{FBS}$, and then the plate was incubated for $24 \mathrm{~h}$ at 37(C. After incubation, tweezers were used to carefully remove the chamber. The upper chamber fluid was drained and approximately $800 \mu \mathrm{l}$ of methanol was added to each well for fixation for $30 \mathrm{~min}$ at room temperature. The chamber was taken out and dyed with $1 \mathrm{ml}$ of $0.5 \%$ crystal violet solution for $30 \mathrm{~min}$. The cells were observed using a microscope (TS100-F, Nikon, Tokyo, Japan). The experimental protocol for the invasion assay of C33A and CaSki cells was similar to that of the migration assay, except for the addition of Matrigel (BD, NJ, USA) and the serum-free medium diluted at a 1:8 ratio at 4 (C. The plate was coated with the upper chamber surface of the bottom membrane in the transwell membrane. The Matrigel was polymerized to a gel at 37(C for $30 \mathrm{~min}$.

\section{3-(4,5-dimethylthiazol-2-yl)-2,5-diphenyltetrazolium (MTT) assay}

The logarithmic-phase cells from cell subculture were plated in 96-well plates with $180 \mu \mathrm{l}(5 \times 103$ cells $)$ per well, and the plates were incubated at 37(C overnight. The effect of miR-4454, ABHD2, and NUDT21 on the proliferation of HPV16-positive CaSki cells and HPV16-negative C33A cells was detected at $24 \mathrm{~h}$, $48 \mathrm{~h}$, and $72 \mathrm{~h}$. After the addition of $20 \mu \mathrm{MTT}$ (Beyotime, Shanghai, China) solution ( $5 \mathrm{mg} / \mathrm{ml}$ ) to the cell culture plates of different groups, the plates were incubated for $4 \mathrm{~h}$. After the supernatant was removed, $150 \mu$ l of dimethyl sulfoxide (Beyotime) solution was added to each well and mixed, and the absorbance value of each well was measured at $490 \mathrm{~nm}$.

\section{Flow cytometry assay}

After the C33A and CaSki cells of the different treatment groups were collected, they were centrifuged at $1000 \times g$ for $5 \mathrm{~min}$, and then suspended by phosphate-buffered saline (PBS) and counted. Approximately 
$2 \times 106 \mathrm{cells} / \mathrm{ml}$ were suspended with binding buffer, and then centrifuged at $300 \times g$ for $10 \mathrm{~min} .14$ Subsequently, $5 \mu$ Innexin V-FITC (Solarbio, Beijing, China) was added to $100 \mu$ of the cell suspension and mixed at room temperature for $10 \mathrm{~min}$, followed by the addition of $5 \mu \mathrm{l}$ propidium iodide and mixed at room temperature for $5 \mathrm{~min}$. Finally, the PBS was added to reach a constant volume $(500 \mu \mathrm{l})$, and cell apoptosis was detected by flow cytometry within $1 \mathrm{~h}$.

\section{Reverse transcription-quantitative PCR (RT-qPCR) assay}

CaSki and C33A cells were harvested and the total RNA was extracted using Trizol reagent (Ambion, TX, USA). The total RNA was reverse-transcribed to cDNA by PrimeScript ${ }^{\text {TM }}$ RT Master Mix (TaKaRa, Dalian, China) according to the manufacturer's instructions. The mRNA was quantified by SYBR ${ }^{\circ}$ Premix Ex

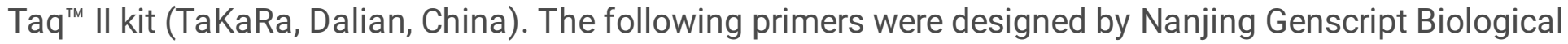
Technology Co., Ltd.: miR-4454F:5'-GGGGGATCCGAGT CA-3',R:5'-AACTGGTGTCGTGGAGTCGGC3';ABHD2F:5'-CGTTGACTACGCCCAGAA-3',R:5'-AAGCCCACGACGACCAG-3';NUDT21F:5'AAACTACCTGGTGGTGAACT-3', R:5'-CTTAGGCTTTGTAATATGTGC-3';U6 F:5'-CTCGCTTCGGC AGCACA-3', R:5'-AACGCTTCACGAATTTGCGT-3'; $\beta$-actin F: 5'-ACACTGTG CCCATCTACG-3', R:5'-

TGTCACGCACGATTTCC-3'. The experiment was executed independently in three biological replicates. General thermal cycling conditions for PCR: $95^{\circ} \mathrm{C}, 3 \mathrm{~min} ; 95^{\circ} \mathrm{C}, 5 \mathrm{sec} ; 56^{\circ} \mathrm{C}, 10 \mathrm{sec} ; 72{ }^{\circ} \mathrm{C}, 25 \mathrm{sec} ;(39$ cycles) $65^{\circ} \mathrm{C}, 5 \mathrm{sec} ; 95^{\circ} \mathrm{C}, 50 \mathrm{sec}$.

\section{Dual luciferase reporter gene assay}

The 3' untranslated regions (UTRs) of the ABHD2 and NUDT21 genes were cloned and amplified in 293 cells. Mutations of the $3^{\prime}$ UTR of the ABHD2 and NUDT21 genes were generated by the QuickChange SiteDirected Mutagenesis kit (Stratagene, CA, USA) at the putative binding site of miR-4454. The wild and mutant ABHD2/NUDT21 genes were cloned into the pmirGLO-vector (Promega, WI, USA) and then cotransfected with pmirGLO-3' UTR-ABHD2 (50 ng) or pmirGIO-3' UTR-NUDT21 (50 ng) and miR-4454 (50 nM) using Lipofectamine ${ }^{\circledR} 2000$ (Invitrogen, MA, USA). The reporter gene plasmid and transcription factor expression plasmid-co-transfected 293 cells were seeded in 24-well plates and incubated for $12 \mathrm{~h}$. After transfection, cell lysates were prepared for $48 \mathrm{~h}$ using Passive Lysis Buffer (Promega, WI, USA). The dual luciferase activity was then examined using the Luciferase Assay System (Promega, WI, USA).

\section{Statistical analysis}

All data were analyzed by the data analysis software SPSS19.0 (SPSS, IL, USA), and are presented as the mean \pm standard deviation. Data were analyzed by one-way analysis of variance followed by Dunnett's or Duncan's test. A $P$-value less than 0.05 was considered statistically significant.

\section{Results}

miR-4454 promotes the invasion, migration, proliferation, and suppresses apoptosis of HPV16 E6/E7positive CaSki cell 
The differentially expressed gene miR-4454 was selected by sequencing in HPV16 E6/E7 positive CaSki cell (Figure 1A-C). The sequencing result showed 23 underexpressed miRNAs $(p<0.05)$ and 4 overexpressed miRNAs $(p<0.05)$ in HPV16 E6/E7 positive cancer cell CaSki compared with HPV16 E6/E7 negative cancer cell. Among these 4 overexpressed, we found the largest fold change for miR-4454 (log2Fold Change=2.8) (Figure 1D). In the present study, miR-4454 levels were confirmed by RT-qPCR (Figure 1E). The results showed that the HPV16 E6/E7 positive cell CaSki expressed higher levels of miR4454 than HPV16 E6/E7 negative cell C33A. Then CaSki and C33A cells were treated with the miR-4454 mimic/inhibitor to detect the effect on the proliferation, apoptosis, migration, and invasion. The results revealed that miR-4454 mimics inhibited the apoptosis of CaSki $(P<0.05)$ and C33A $(P<0.05)$ cells (Figure 1F-G), and promoted the proliferation of CaSki $(P<0.001)$ and C33A $(P<0.001)$ cells (Figure $1 \mathrm{H})$. Although miR-4454 mimics significantly increased the proliferation of CaSki cells, there was no significant difference in the effect between CaSki and C33A cells. In addition, miR-4454 mimics significantly promoted the invasion (Figure 2A-B) $(P<0.05)$ and migration (Figure 2C-D) $(P<0.05)$ of both CaSki and C33A cells, and the effect was significantly greater in CaSki cells compared to C33A cells $(P<0.05)$. These data suggested that miR-4454 can increase the invasion and migration of HPV16 E6, E7-positive cells.

\section{miR-4454 targets ABHD2 and NUDT21}

The interactions between miR-4454 and ABHD2/NUDT21 were evaluated in 293 cells by a dual luciferase assay (Figure2Eख2H). In the $A B H D 2$ wild-type (WT) group, R/F was significantly increased in the miR4454-transfected cells compared with the control $(P<0.001)$ and miR-4454 negative control (NC)transfected cells $(P<0.01)$, while there was no significant difference in $A B H D 2$ mutated (Mut) group $(P<$ 0.001). Similarly, in the NUDT21 WT group, F/R was significantly decreased in the miR-4454-transfected cells compared with the control $(P<0.01)$ and miR-4454 NC-transfected cells $(P<0.01)$, while there was no significant difference in NUDT21 mutated (Mut) group $(P<0.001)$. These results suggested that miR4454 targets both $A B H D 2$ and NUDT21. To validate these results, an RT-qPCR assay and westen blot were used to detect the expression of $A B H D 2$ and NUDT21 mRNA levels and protein levels in CaSki and C33A cells (Figure2F-2I,2K-2N). Both of the targets were down-regulated in cells transfected with the miR-4454 mimics $(P<0.05 / P<0.01)$ and were up-regulated in cells transfected with the miR-4454 inhibitor $(P<$ $0.05)$ compared with the control. Moreover, compared with C33A cells, the expression levels of $A B H D 2$ and NUDT21 were markedly decreased in CaSki cells $(P<0.05)$ (Figure2G $\llbracket 2 \mathrm{~J})$.

\section{ABHD2 and NUDT21 suppresses the invasion and migration of HPV16 E6/E7-positive CaSki cells}

Since we demonstrated that $A B H D 2$ and NUDT21 are targets of miR-4454, we next explored the effects of $A B H D 2$ and NUDT21 on the invasion and migration of HPV16-positive cells (CaSki) and HPV16-negative cells (C33A). The RT-qPCR assay confirmed the successful construction of pre-ABHD2, sh-ABHD2, preNUDT21, and sh-NUDT21 plasmids. The expression of $A B H D 2$ was up-regulated in pre-ABHD2 group and was down-regulated in sh-ABHD2 group (Figure $3 A$ ). In addition, the expression of NUDT21 was upregulated in pre-NUDT21 group and down-regulated in sh-NUDT21 group (Figure 3B). The invasion and 
migration of the transfected cells were then detected by a transwell assay. Our results showed that preABHD2 markedly decreased the invasion (Figure 3C-D) and migration (Figure 3E-F) of both C33A and CaSki cells $(P<0.05)$, whereas sh-ABHD2 significantly increase the invasion and migration of C33A and CaSki cells $(P<0.05$ and $P<0.01$, respectively). These data indicated that $A B H D 2$ can decrease the invasion and migration of HPV16 cells (C33A and CaSki), or in other words, a lack of ABHD2 can increase the invasion and migration of HPV16 cells (C33A and CaSki). Moreover, compared with C33A cells, the invasion and migration were promoted to a greater extent in CaSki cells $(P<0.05)$. Similar results were obtained for pre-NUDT21 and sh-NUDT21 (Figure 4). Overall, these results suggest that HPV16 E6/E7 can affect cell invasion and migration via regulating miR-4454 targeting ABHD2/NUDT21.

\section{Discussion}

High-risk HPV16 E6/E7 can regulate the tumor suppressor p53 and pRb, respectively 1919 . In tumor necrosis factor-alpha-stimulated human cervical cancer HeLa cells, miR-4454 was identified as an NF-KBtargeting miRNA, with roles in the anti-inflammatory, apoptosis, and other physiological processes of cervical cancer cells 18 . A previous study showed that miR-4454 was overexpressed in the bladder tumor, WBCs, and urine17 . miR-4454 is involved in promoting the inflammation, catabolism, and cell death activity of chondrocytes in spinal osteoarthritis (facet joints) 15-17. This activation consequently promotes the expression of $\mathrm{c}-\mathrm{Myc}$, and cell cycle protein $\mathrm{A}$ and $\mathrm{E}$ (CyclinA/E), which is beneficial to the synthesis of DNA and induces the progression of cells from $\mathrm{G} 1$ to $\mathrm{S}$, thus promoting cell proliferation.20 They suggested that miR-4454 may affect cancer cell migration, invasion, proliferation, and apoptosis. In the present study, we determined specific high expression of miR-4454 in HPV16 E6/E7 through a gene chip screening process. Therefore, we focused on the effects of miR-4454 and its downstream targets determined by querying the miRNAs target database on cervical cancer cells. Our data are in line with previous studies showing that miR-4454 is overexpressed in tumors, and is highly enriched in the HPV16 E6/E7 positive cervical cancer cell. Moreover, miR-4454 promoted the proliferation, invasion, and migration, and inhibited the apoptosis of CaSki cell compared with C33A cell.

The subsequent study was interested in the target of miR-4454 to further understand its effect on the progression of cervical cancer. ABHD2 and NUDT21 were selected as candidate targets of miR-4454 based on information from online databases. Yun et al. 23 showed that the expression of $A B H D 2$ was upregulated by androgen in $\mathrm{LNCaP}$ and $\mathrm{VCaP}$ cells. Disruption of $A B H D 2$ expression also promotes cell proliferation and migration of $\mathrm{LNCaP}$ cells. According to these results, $A B H D 2$ was proposed as a novel target for the diagnosis and treatment of prostate cancer. 22 showed that down-regulation of $A B H D 2$ could promote a malignant phenotype and was associated with a poor prognosis for women with serous ovarian cancer. In addition, Obinata et al. 21 described a novel mechanism by which the serine hydrolase $A B H D 2$ regulates calcium transfer from the endoplasmic reticulum to the mitochondria to affect cell death (anoikis) resistance, which is related to malignant phenotypes and is a hallmark of cancer. Yamanoi et al. 
In our study, we demonstrated that $A B H D 2$ is a target of carcinogenic miR-4454 and its expression is negatively correlated to that of miR-4454. Therefore, we supposed that suppression of $A B H D 2$ would have a carcinogenic effect. The invasion and migration of HPV16 E6/E7-positive CaSki cell were increased to a greater extent under $A B H D 2$ inhibition compared with the effects observed in HPV16negative C33A cells, consistent with the previous study in prostate cancer 24 . Our data also suggested that NUDT21 is negatively correlated to miR-4454, and knockdown of NUDT21 increased the invasion and migration of HPV16 E6/E7-positive CaSki cell compared with HPV16-negative C33A cell, indicating an effect in promoting the development of human cervical cancer. 23 . The other study showed that knockout of NUDT21 promoted the proliferation, metastasis, and tumorigenesis of HCC cells, whereas enhanced the expression of NUDT21 had the opposite effect

In conclusion, miR-4454 was overexpressed in HPV16 positive cervical cancer cells, and HPV16 E6/E7 may affect cancer cell invasion and migration via miR-4454/ABHD2/NUDT21 axis in cervical cancer cells. In follow-up experiments, we will further explore the specific roles of $A B H D 2 / N U D T 21$ in the development of HPV16-positive cervical cancer and its related mechanisms. This work is expected to provide new insights into the mechanism by which HPV16 E6/E7 regulate ABHD2/NUDT21 through miR-4454 to better understand the malignant transformation of cervical cancer.

\section{Declarations}

\section{Acknowledgements}

This work was supported by Open Research Fund Program of the State Key Laboratory of Virology of China (2016KF004), Research Fund of Hubei Province Public Health Bureau (WJ2015MB144) and Research Fund of Wuhan Public Health Bureau (WX15A12).

\section{Authors' contributions}

$\mathrm{HW}, \mathrm{SL}, \mathrm{HH}$ and ZL carried out the molecular biology analysis, participated in the design of the study and the clinical specimen collection, and drafted the manuscript. WW, MZ, JW and YL carried out the clinical specimen collection, participated in the data analysis, and performed the statistical analysis. ZL and HW conceived of and designed the study, and participated in the data analysis and coordination, and helped to draft the manuscript. All authors read and approved the final manuscript.

\section{Competing interests}

The authors declare that they have no competing interests.

\section{Availability of data and materials}

The datasets used and/or analyzed during the current study are available from the corresponding author on reasonable request. 


\section{References}

1. Lu, Q, Ma, D,Zhao, S(2012). DNA methylation changes in cervical cancers. Methods in molecular biology 863:155-176.

2. Robova H, Rob L, Halaska MJ,Pluta M, Skapa P,Strnad P,et al(2013). High-dose density neoadjuvant chemotherapy in bulky ib cervical cancer. Gynecol Oncol 128:49-53.

3. Carozzi FM, Burroni E, Bisanzi S,Puliti D, Confortini M,Giorgi Rossi P,et al(2011). Comparison of clinical performance of abbott realtime high risk hpv test with that of hybrid capture 2 assay in a screening setting. J Clin Microbiol 49:1446-51.

4. Eiben GL, da Silva DM,Fausch SC, Le Poole IC,Nishimura Ml,et al(2003). Cervical cancer vaccines: Recent advances in hpv research. Viral Immunol 16:111-21.

5. Yang WW, Wang ZG, Chen GF, Shi JF, Chen J, Ma QH,et al(2018). Comparison of a newly developed hpv genotyping assay (mojin hpv kit) with cobas $4800 \mathrm{hpv}$ test for detection of high-risk hpv in specimens collected in surepath solution. Clin Lab 64:387-391.

6. Arbyn M, Xu L, Simoens C, Martin-Hirsch PP(2018). Prophylactic vaccination against human papillomaviruses to prevent cervical cancer and its precursors. Cochrane Database Syst Rev 5:CD009069.

7. Wu MZ, Li WN, Cha N, Tian LX, Zhang YI, Wu X,et al(2018). Diagnostic utility of hpv16 e6 mrna or e7 mrna quantitative expression for cervical cells of patients with dysplasia and carcinoma. Cell Transplant 27:1401-1406.

8. Petry KU(2014). Hpv and cervical cancer. Scand J Clin Lab Invest Suppl 244:59-62.

9. Ghittoni R, Accardi R, Hasan U, Gheit T, Sylla B, Tommasino M(2010). Virus Genes 40:1- 13.

10. Lace MJ, Anson JR,Haugen TH, Turek LP(2010). Interferon regulatory factor (irf)-2 activates the hpv16 e6-e7 promoter in keratinocytes.Virology 399:270-9.

11. Charette ST, McCance DJ(2007).The e7 protein from human papillomavirus type 16 enhances keratinocyte migration in an akt-dependent manner. Oncogene 26:7386-90.

12. Hellner K, Mar J, Fang F, Quackenbush J, Munger K(2009). Hpv16 e7 oncogene expression in normal human epithelial cells causes molecular changes indicative of an epithelial to mesenchymal transition. Virology 391:57-63.

13. Santarelli L, Gaetani S, Monaco F, Bracci M(2018). Four-mirna signature to identify asbestos-related lung malignancies. Cancer Epidemiol Biomarkers Prev doi: 10.1158/1055-9965.EPI-18-0453. [Epub ahead of print] 
14. Priyadarssini M, Chandrashekar L, Rajappa M(2018). Effect of methotrexate monotherapy on t-cell subsets in the peripheral circulation in psoriasis. Clin Exp Dermatol doi: 10.1111/ced.13795. [Epub ahead of print] .

15. Wang X, Meyers C, Guo M, Zheng ZM(2011). Upregulation of p18ink4c expression by oncogenic hpv e6 via p53-mir-34a pathway. Int J Cancer 129:1362-72.

16. Suzuki HI, Miyazono K(2013). P53 actions on microrna expression and maturation pathway. Methods Mol Biol 962:165-81.

17. Garcia-Garcia E,Pino-Barrio MJ, Lopez-Medina L, Martinez-Serrano A(2012). Intermediate progenitors are increased by lengthening of the cell cycle through calcium signaling and p53 expression in human neural progenitors. Mol Biol Cell 23:1167-80.

18. Nakamura A, Rampersaud YR(2016). Identification of microrna-181a-5p and microrna-4454 as mediators of facet cartilage degeneration. JCI Insight 1:e86820.

19. Armstrong DA, Green BB, Seigne JD, Schned AR, Marsit CJ(2015). Microrna molecular profiling from matched tumor and bio-fluids in bladder cancer. Mol Cancer 14:194.

20. Ji H,Chen M, Greening DW, He W,Rai A, Zhang W, et al(2014). Deep sequencing of rna from three different extracellular vesicle (ev) subtypes released from the human lim1863 colon cancer cell line uncovers distinct mirna-enrichment signatures. PLoS One 9:e110314.

21. Yun B, Lee H,Powell R, Reisdorph N, Ewing H, Gelb M.H,et al(2017). Regulation of calcium release from the endoplasmic reticulum by the serine hydrolase abhd2. Biochem Biophys Res Commun 490:1226-1231.

22. Yamanoi K,Matsumura N, Murphy SK, Baba T, Abiko K,Hamanishi J,et al(2016). Suppression of abhd2, identified through a functional genomics screen, causes anoikis resistance, chemoresistance and poor prognosis in ovarian cancer. Oncotarget 7:47620-47636.

23. Obinata D,Takada S, Takayama K, Urano T, Ito A,Ashikari D, et al(2016). Abhydrolase domain containing 2, an androgen target gene, promotes prostate cancer cell proliferation and migration. Eur $\mathrm{J}$ Cancer 57:39-49.

24. Tan S,Li H,Zhang W, Shao Y,Liu Y, Guan H, et al(2018). Nudt21 negatively regulates psmb2 and cxxc5 by alternative polyadenylation and contributes to hepatocellular carcinoma suppression. Oncogene 37:4887-4900.

\section{Figures}


$\mathbf{A}$
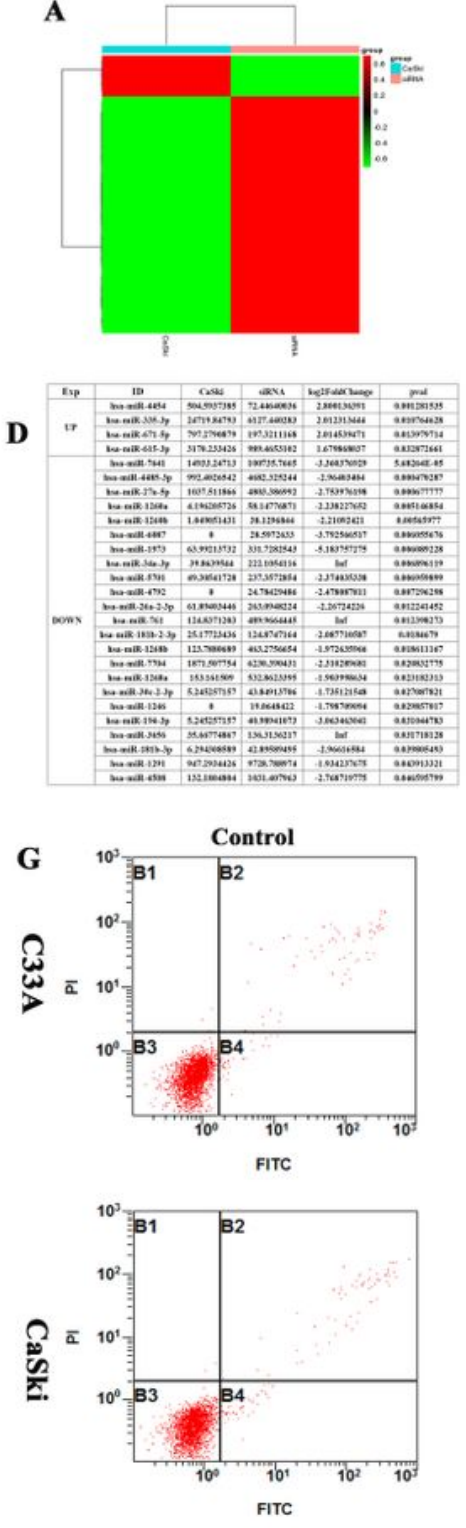

H

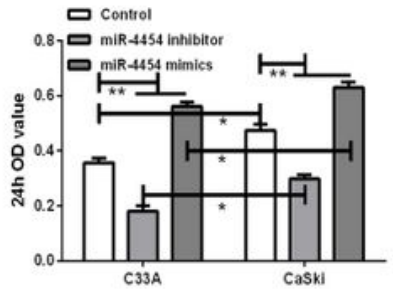

B

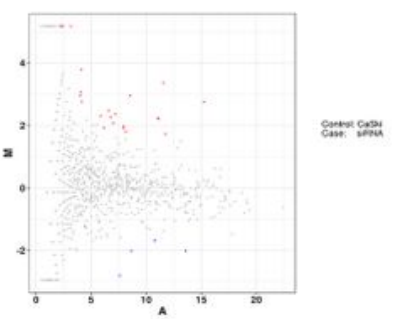

E
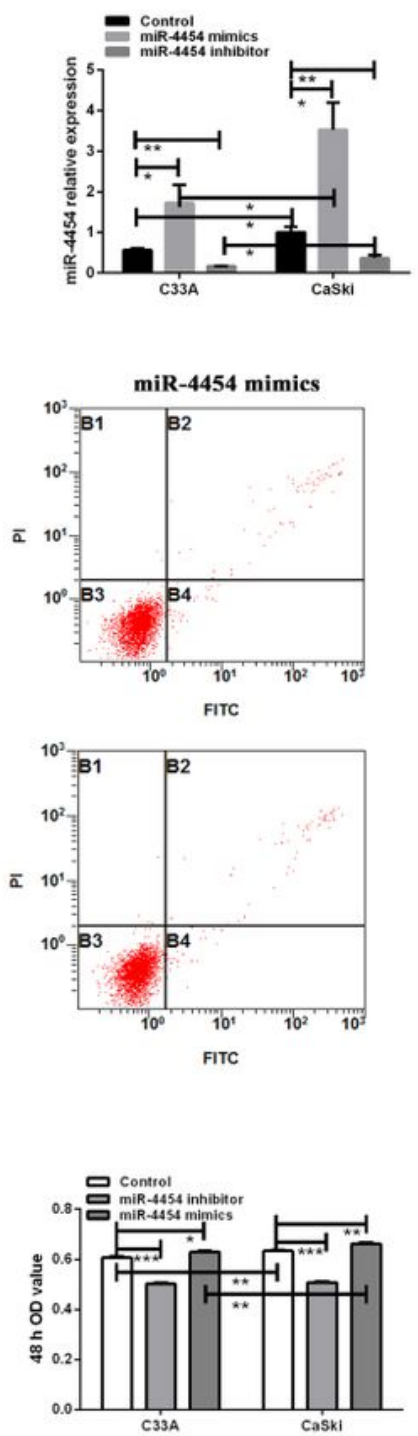

C

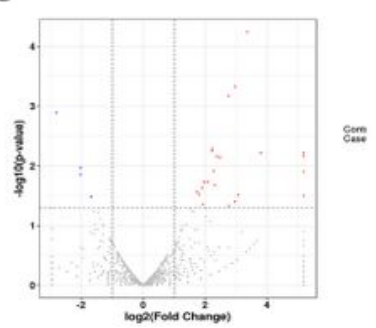

F
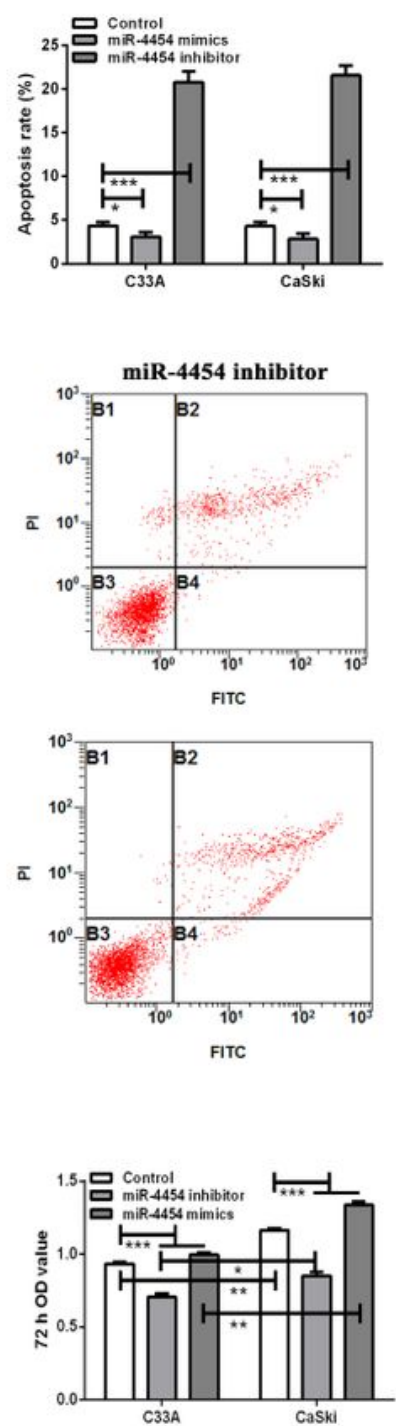

\section{Figure 1}

miR-4454 was highly expressed in HPV16 E6/E7 positive CaSki cell. (A) The thermal map result for sequencing in CaSki cell.(B-C)The volcano map result for sequencing in CaSki cell.(D)The bioinformatics analysis for dramatically up and down regulated miRNAs in CaSki cell.(E)The expression of miR-4454 was detected by RT-qPCR in HPV16-positive cells (CaSki) and HPV16-negative cells (C33A) transfected with miR-4454 mimics and miR-4454 inhibitor.(F)Statistical analysis of the apoptosis rate in miR-4454 
mimics- and miR-4454 inhibitor-transfected CaSki and C33A cells.(G)Flow cytometry images of cell apoptosis rates. $(\mathrm{H})$ The proliferation of CaSki and C33A cells transfected with miR-4454 mimics and miR-4454 inhibitor at $24 \mathrm{~h}, 48 \mathrm{~h}$, and $72 \mathrm{~h}$. All data were obtained in triplicate; *, **, and $* \star \star$ indicate $\mathrm{P}<$ $0.05, P<0.01$, and $P<0.001$, respectively.
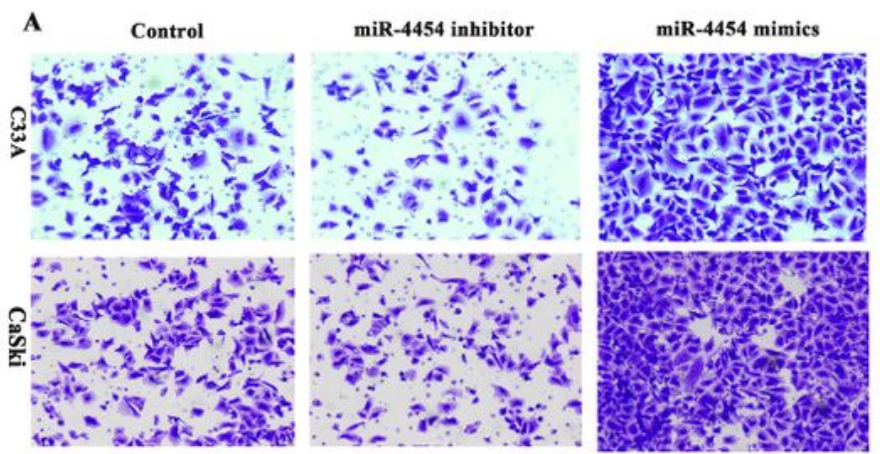

B
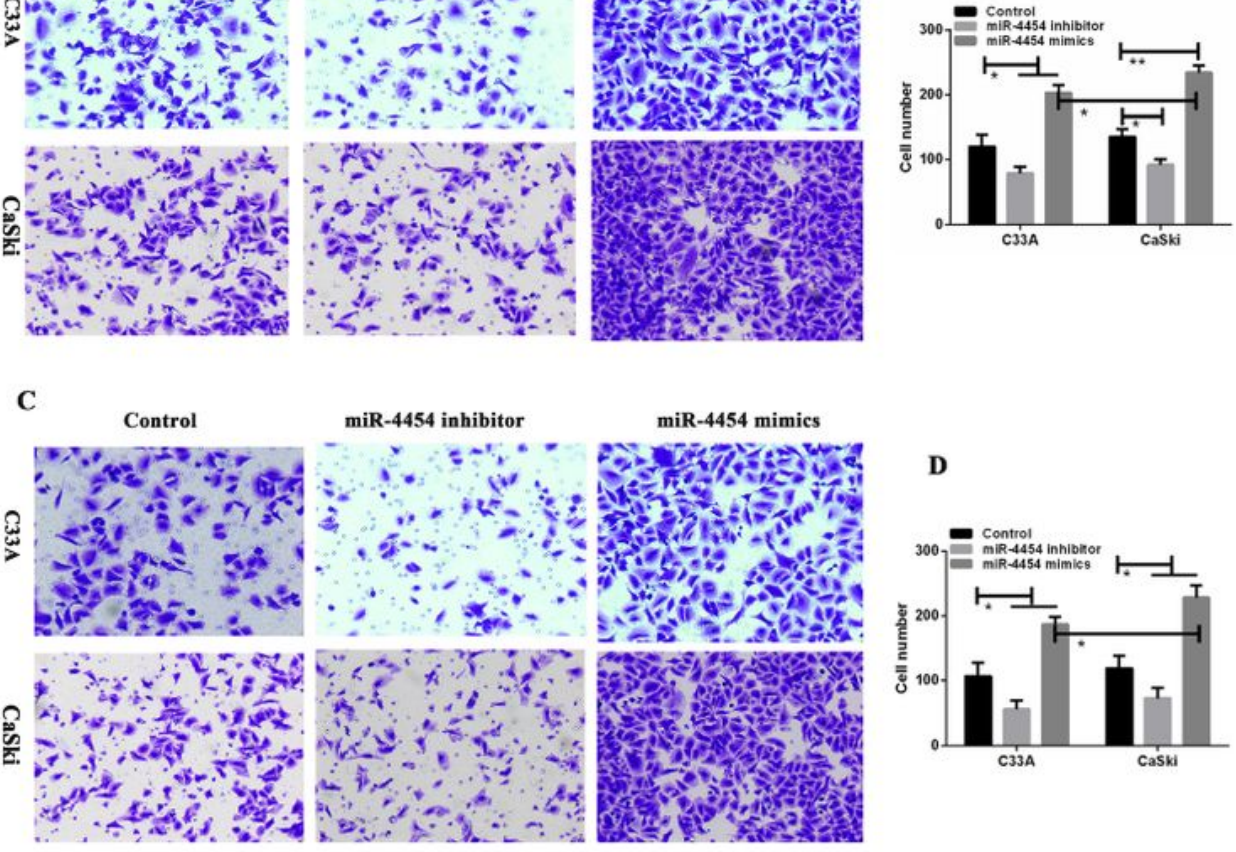

$\mathbf{E}$

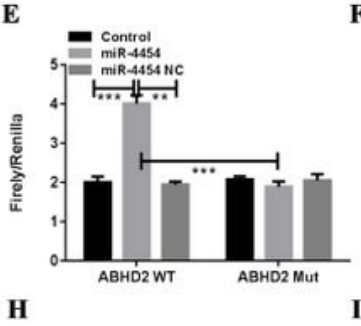

H

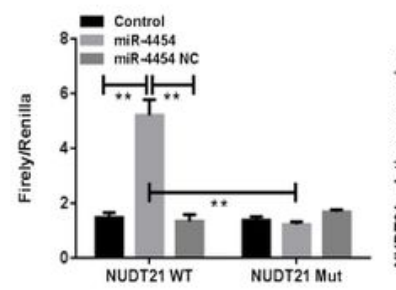

$\mathbf{F}$

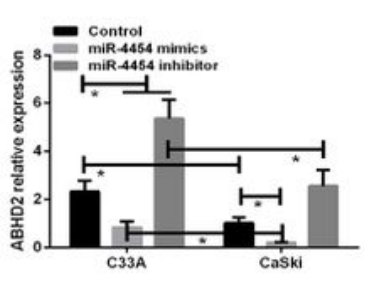

I

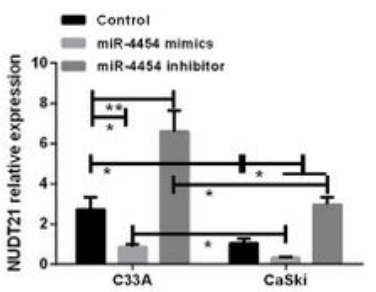

G

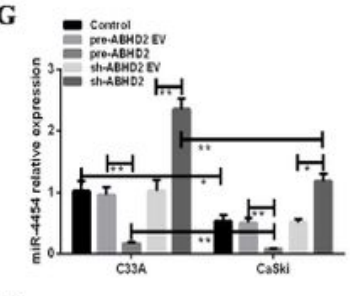

$\mathbf{J}$

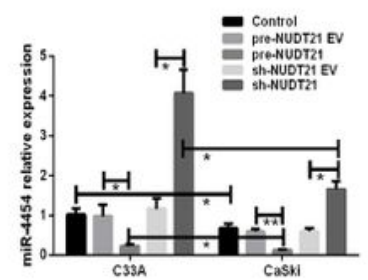

K

L

M

N
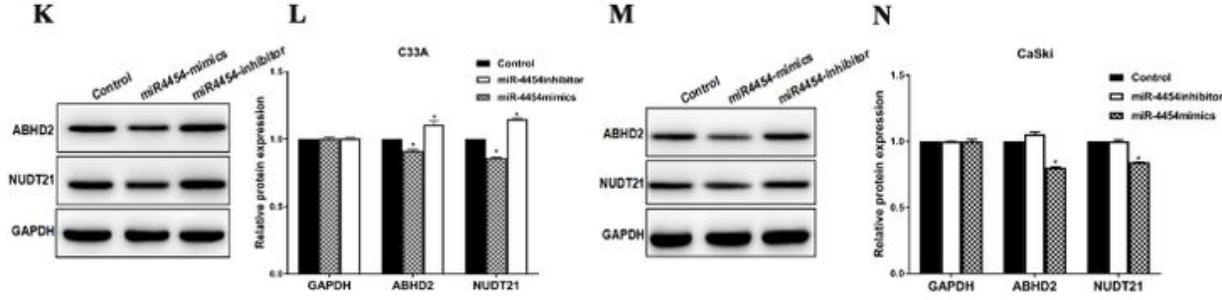

Figure 2 
miR-4454 affected the invasion and migration on HPV16 E6/E7-positive CaSki cells. (A) Transwell assay was used to detect cell invasion in CaSki and C33A cells. (B) Statistical analysis of the invasion of human cervical cancer cells (CaSki and C33A) treated with miR-4454 mimics and miR-4454 inhibitor. (C)Transwell assay was used to detect cell migration in CaSki and C33A cells. (D)Statistical analysis of migration in human cervical cancer cells (CaSki and C33A) treated with miR-4454 mimics and miR-4454 inhibitor. All data were obtained in triplicate; ${ }^{*}$, ${ }^{*}$, and $* \star \star$ indicate $\mathrm{P}<0.05, \mathrm{P}<0.01$, and $\mathrm{P}<0.001$, respectively.(E)Interactions between miR-4454 and ABHD2 evaluated in 293 cells by a dual luciferase assay.(F)Relative mRNA expression levels of ABHD2 in cells transfected with the miR-4454 mimics and miR-4454 inhibitor. (G)Relative expression levels of miR-4454 in cells transfected with the pre-ABHD2 and sh-ABHD2.(H)Interactions between miR-4454 and NUDT21 evaluated in 293 cells by a dual luciferase assay.(I)Relative mRNA expression levels of NUDT21 in cells transfected with the miR-4454 mimics and miR-4454 inhibitor. $(\mathrm{J})$ Relative expression levels of miR-4454 in cells transfected with the pre-NUDT21 and sh-NUDT21.(K)Western blot analyses of ABHD2 and NUDT21 in C33A cells transfected with miR4454 mimics and miR-4454 inhibitor.(L) Relative protein expression levels of NUDT21 and ABHD2 in C33A cells transfected with the miR-4454 mimics and miR-4454 inhibitor.(M)Western blot analyses of ABHD2 and NUDT2 1 in CaSki cells transfected with miR-4454 mimics and miR-4454 inhibitor.(N)Relative protein expression levels of NUDT21 and ABHD2 in CaSki cells transfected with the miR-4454 mimics and miR-4454 inhibitor.data were obtained in triplicate; * $* *$, and $* \star \star$ indicate $P<0.05, \mathrm{P}<0.01$, and $\mathrm{P}<$ 0.001 , respectively. 
A

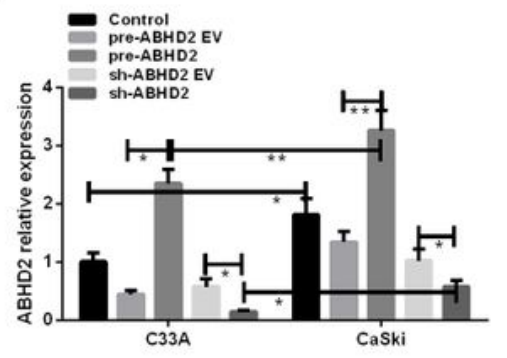

C

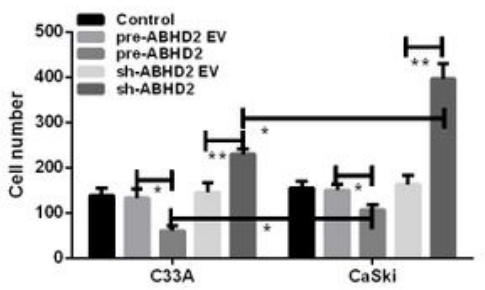

B

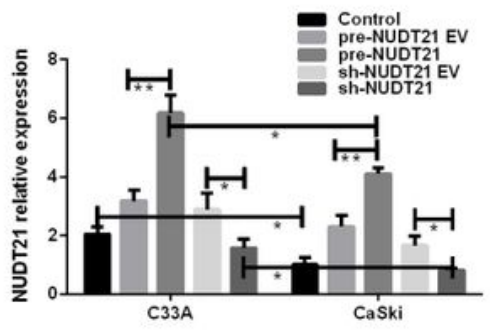

E

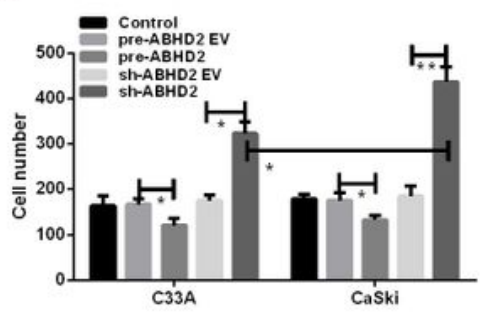

D
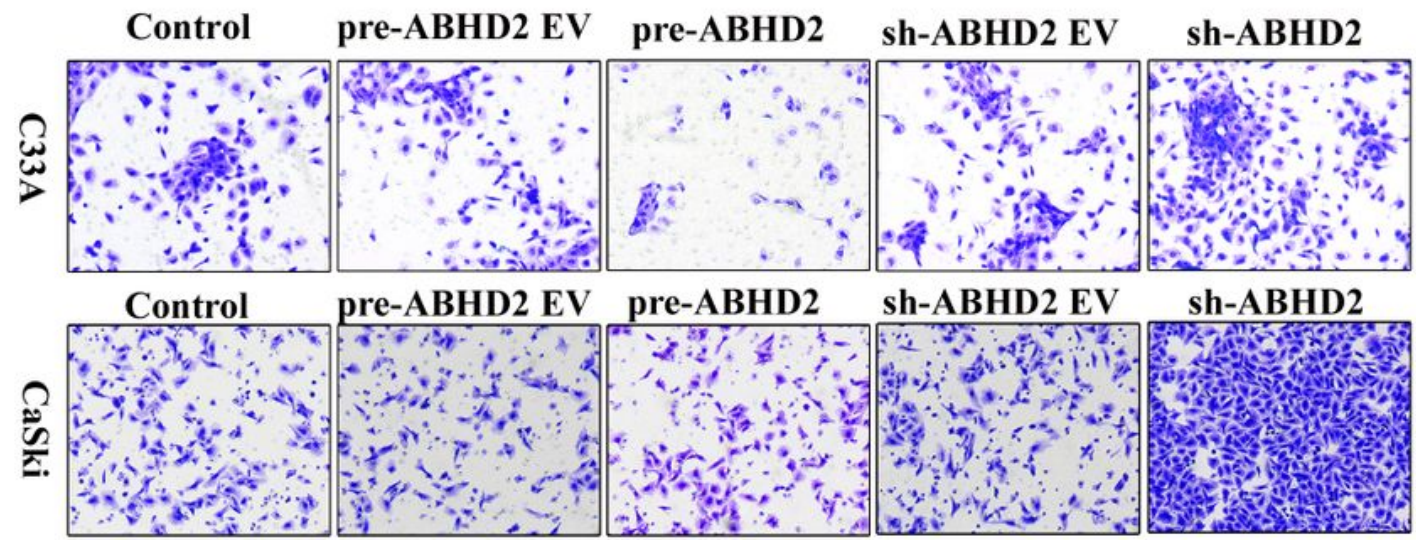

F
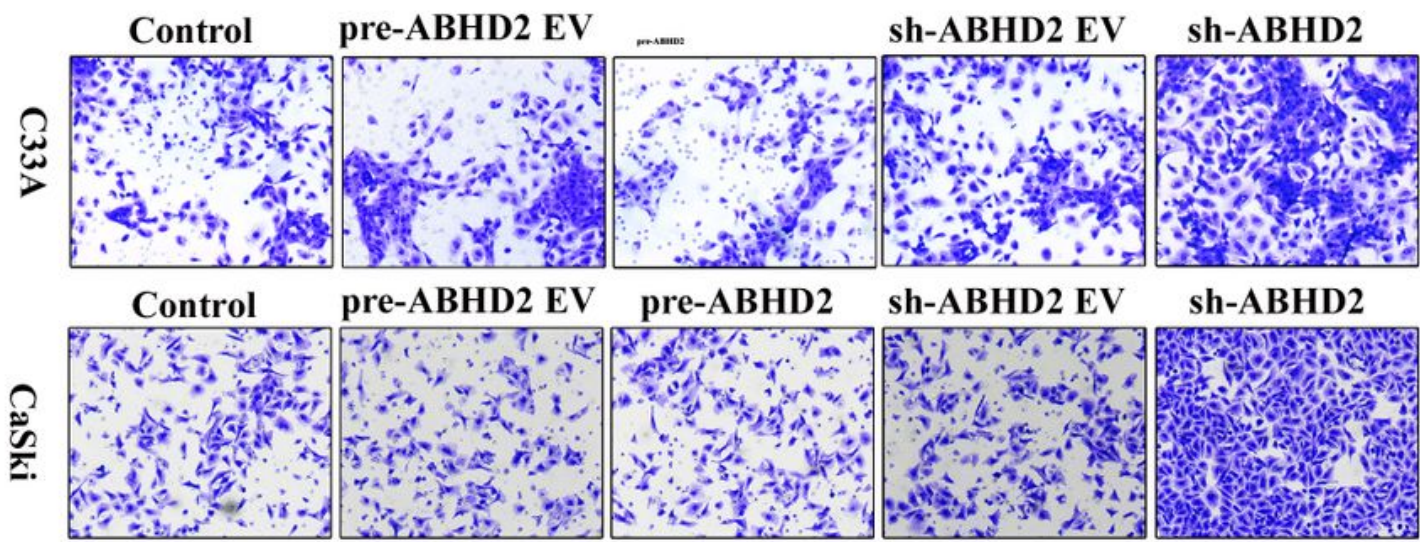

Figure 3

ABHD2 affects the invasion and migration of HPV16 E6/E7-positive CaSki cells. (A) Construction of preABHD2 and sh-ABHD2 confirmed by RT-qPCR. (B) Construction of pre-NUDT21 and sh-NUDT21 confirmed by RT-qPCR. (C) Statistical analysis of invasion in human cervical cancer cells (CaSki and C33A) treated with pre-ABHD2 and sh-ABHD2. (D) Transwell pictures of cell invasion in CaSki and C33A cells. (E) Statistical analysis of migration in human cervical cancer cells (CaSki and C33A) treated with pre-ABHD2 
and sh-ABHD2. (F) Transwell assay was used to detect cell migration in CaSki and C33A cells. All data were obtained in triplicate; *, **, and $* \star *$ indicate $\mathrm{P}<0.05, \mathrm{P}<0.01$, and $\mathrm{P}<0.001$, respectively.

A

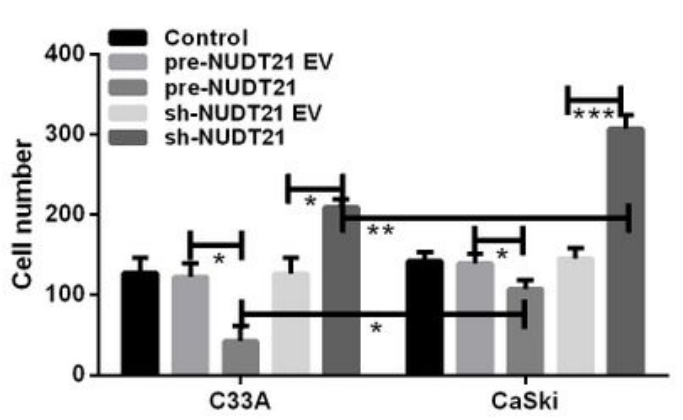

C

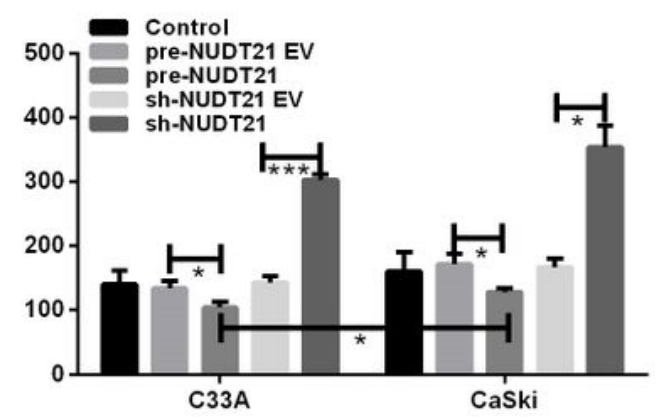

B

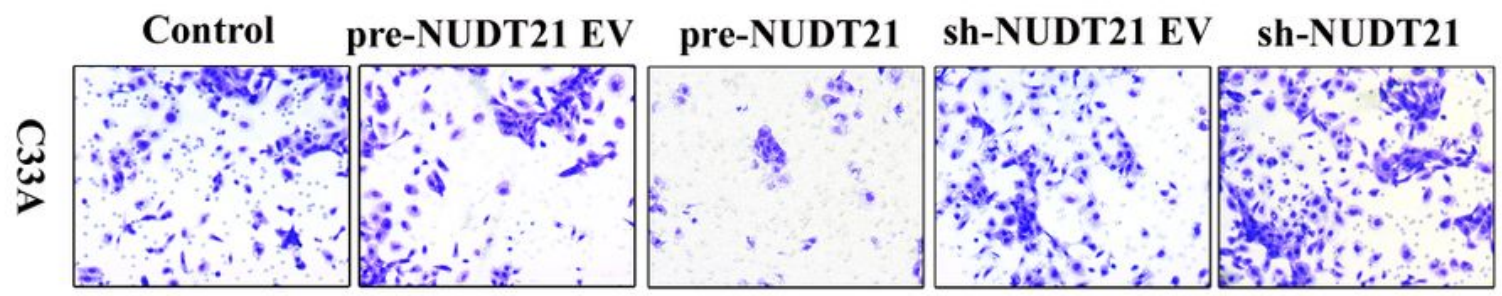

Control pre-NUDT21 EV pre-NUDT21 sh-NUDT21 EV sh-NUDT21
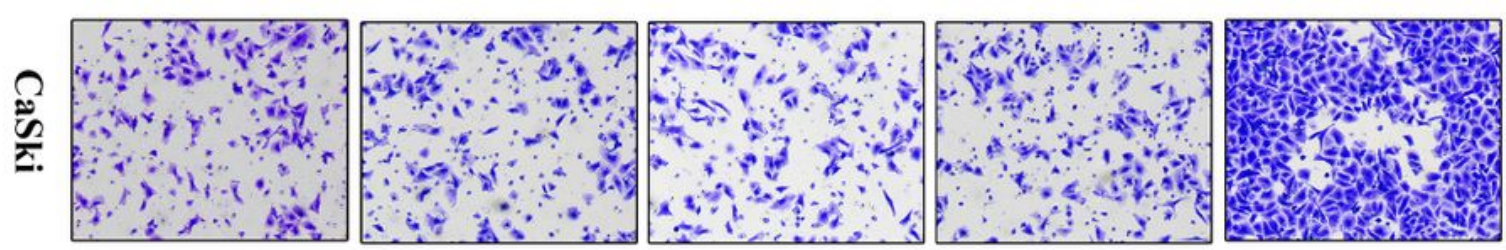

D
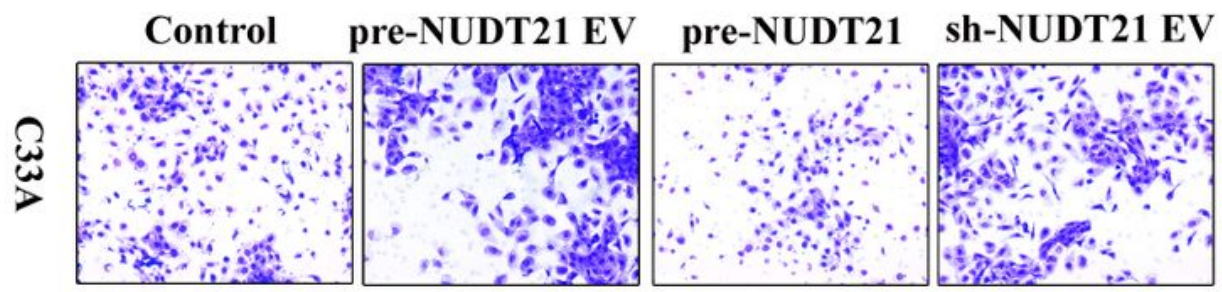

sh-NUDT21

\section{Control}
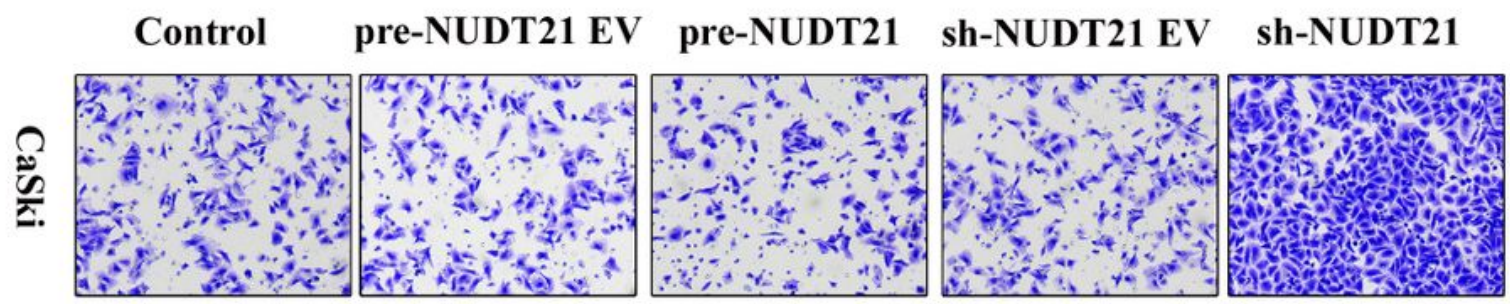

Figure 4

NUDT21 affects the invasion and migration of HPV16 E6/E7-positive CaSki cells. (A) Statistical analysis of invasion in human cervical cancer cells (CaSki and C33A) treated with pre-NUDT21 and sh-NUDT21.

(B) Transwell assay was used to detect cell invasion in CaSki and C33A cells. (C) Statistical analysis of 
migration in human cervical cancer cells (CaSki and C33A) treated with pre- NUDT21 and sh-NUDT21. (D) Transwell assay was used to detect cell migration in CaSki and C33A cells. All data were obtained in triplicate; * ${ }^{* \star}$, and $* \star \star$ indicate $P<0.05, P<0.01$, and $P<0.001$, respectively. 